\title{
A Theoretical Explanation on Gell-Mann-Nishijima Formula with Singular Number and the Establishment of Lepton Quantum Number Constrained Equation
}

\author{
Hua Ma \\ College of Science, Air Force University of Engineering, Xi'an, People's Republic of China
}

\section{Email address:}

mahuar@xjtu.edu.cn

\section{To cite this article:}

Hua Ma. A Theoretical Explanation on Gell-Mann-Nishijima Formula with Singular Number and the Establishment of Lepton Quantum Number Constrained Equation. American Journal of Modern Physics. Vol. 9, No. 6, 2020, pp. 84-90. doi: 10.11648/j.ajmp.20200906.12

Received: November 11, 2020; Accepted: December 1, 2020; Published: December 8, 2020

\begin{abstract}
In this paper, two fundamental problems of particle physics are studied theoretically. The first one is: to solve the problem of establishing general quantum number constrained equation, the symmetry transformation mechanism of charge eigenstates for elementary particles is adopted, and the general quantum number constrained equations are established, which are applicable to physical particles. For hadrons, an equation is completely consistent with Gell-Mann-Nishijima formula. For leptons, the lepton quantum numbers are exactly the solutions of an equation, which is just the lepton quantum number constrained equation. The second problem is: to solve the problem of understanding singularity and calculating singular numbers, a hypothesis that a composite particle may has virtual structure is proposed. According to this hypothesis, the singular particles must be composite particles, and have virtual structures. In a virtual structure, the particles and antiparticles of component particles can form particle-antiparticle pairs, which have great influence such as improving mass and changing life of composite particles. Therefore, the composite particle with particle-antiparticle pairs in its virtual structure is singular particle, and the singular number is the number of particle-antiparticle pairs. These two theoretical results are in good agreement with the already achieved experimental results of particle physics, can explain the related phenomena of physical particles from a deeper physical mechanism, and theoretically predict the existence of singular leptons and several new singular hadrons.
\end{abstract}

Keywords: Particle Physics, Symmetric Transformation, Quantum Number, Elementary Particle, Singular Particle, Singular Number, Hadron, Lepton

\section{Introduction}

In the in-depth exploration of particle physics, the continuous development of particle accelerators, detection means, data processing and computing technology has not only brought about the progress of particle physics, but also promoted the development of the whole science and technology [1-7]. The establishment of the standard model of particle physics is one of the important achievements of physics in the 20th century. It can describe the properties of quark, lepton, photon, gluon, intermediate boson and Higgs particle, as well as the three basic interactions [8-10]. At present, there are many research directions in particle physics, such as hadron physics, heavy flavor physics, lepton physics, neutrino physics, accurate test of standard model, symmetry and symmetry destruction, standard model expansion, etc. these works have even played an important role in the study of the evolution of the universe [11-14].

At deeper levels of physical mechanism, are there theoretical problems need to be more in-depth thought in particle physics? For instance, Gell-Mann-Nishijima formula is a very successful quantum number constrained equation for hadrons, it refers to the relationship between hadron charge, isospin third component, baryon number and singular number, but cannot be used to describe the quantum number constrained equation of leptons. In order to describe the properties of singular sub, singular number is also introduced into the formula [15-18]. Inspired by this formula, some problems need to be considered are put forward: What is the deeper physical mechanism on which the formula is established? How to establish the general quantum number constrained equations, through which hadrons and leptons 
both can be described? The baryon numbers of all hadrons can be summed, which shows that hadrons belong to one class, while leptons are of three classes, why? What is the nature of singular particles? Why are singular numbers not conserved? There are singularities in hadrons, can there be singularities in leptons?

After years of study and thinking, I put forward the solutions to the above problems. (1) About the problems of quantum number constrained equation, the symmetry transformation mechanism focusing on charge eigenstates for physical particles is proposed, thus the quantum number constrained equations of physical particles are established through method of operator transformation. In the field of hadrons, an equation is completely consistent with Gell-Mann-Nishijima formula. In the field of leptons, the quantum numbers of lepton are exactly the solution of an equation, indicating that this equation is the lepton quantum number constrained equation. And based on this equation, the physical phenomena of one kind of baryon number and three kinds of lepton number can be reasonably explained. (2) About the problems of understanding singular particles and calculating singular numbers, a hypothesis that a composite particle may has virtual structure is proposed. According to this hypothesis, the singular particles must be composite particles, and have virtual structures. In a virtual structure, the particles and antiparticles of component particles can form particle-antiparticle pairs, which have great influence such as improving mass and changing life of composite particles. Therefore, the composite particle with particle-antiparticle pairs in its virtual structure is singular particle, and the singular number is the number of particle-antiparticle pairs. These two theoretical results are in good agreement with the already achieved experimental results of particle physics, can explain the existing physical phenomena of particles from a deeper physical mechanism, and theoretically predict the existence of singular leptons and several new singular hadrons. These theoretical results are summarized below, and put forward some ideas for further researches.

\section{Quantum Number Constrained Equation of Physical Particle}

\subsection{Equation Derivation Based on Symmetry Mechanism}

In particle physics, each kind of particle has multiple quantum numbers. What are the interactions and constraints between these quantum numbers? The effective way is to sum quantum numbers by transformation, thus to establish the quantum number constrained equation of physical particle. In the following, using up-down operator method, choose a basic quantum number as the ground state to make multiple transformations, and accordingly introduce other kinds of quantum numbers with intrinsic conservation, then obtain the summation effect of quantum numbers with associated transformations.

As we all know, charge is the most typical quantum number with conservation, quantum and eigenstate in particle physics
[19-21], so I propose the principle that elementary particles must be at charge eigenstates. Based on this principle, in the analysis of particle quantum numbers, can take the charge number $q$ and charge operator $\widehat{Q}$ as the basic quantum number and basis operator. For elementary particles, the charge number is $q=0, \pm 1$ being the eigenvalues of charge operator, so the charge operator $\widehat{Q}$ and eigenstate $|\varphi\rangle$ must satisfy the following expressions:

$$
\widehat{\mathrm{Q}}|\varphi\rangle=q|\varphi\rangle, \widehat{\mathrm{Q}}^{2}= \pm \widehat{\mathrm{Q}}
$$

In the intrinsic space associated with charges, there are three types of symmetric transformations [22]: inversion, co-spin and rotation. So, use inversion operator $\widehat{A}$ to inverse the initial state $|\varphi\rangle$, use co-spin operator $\hat{B}$ to add co-spin quantum number $c$, and use rotation operator $\widehat{\mathrm{C}}$ to add rotation quantum number $r$. According to the operator theory, these three operators must satisfy the following relationships with charge operator $\widehat{Q}$ :

$$
\widehat{\mathrm{Q}} \widehat{\mathrm{A}}+\widehat{\mathrm{A}} \widehat{\mathrm{Q}}=0, \widehat{\mathrm{Q}} \widehat{\mathrm{B}}-\widehat{\mathrm{B}} \widehat{\mathrm{Q}}=c \widehat{\mathrm{B}}, \widehat{\mathrm{Q}} \widehat{\mathrm{C}}-\widehat{\mathrm{C}} \widehat{\mathrm{Q}}=r \widehat{\mathrm{C}}
$$

Make operator transformations: Firstly, initial state is transformed by $\widehat{\mathrm{A}}$ from $|\varphi\rangle$ to $\widehat{\mathrm{A}}|\varphi\rangle$, accordingly the eigenvalue of charge at new state is changed from $q$ to $-q$, the derivation and description are as follows:

$$
\widehat{\mathrm{Q}} \widehat{\mathrm{A}}|\varphi\rangle+\widehat{\mathrm{A}} \widehat{\mathrm{Q}}|\varphi\rangle=0, \widehat{\mathrm{Q}} \widehat{\mathrm{A}}|\varphi\rangle=-q \widehat{\mathrm{A}}|\varphi\rangle
$$

Then, the second state is transformed by $\widehat{B}$ from $\widehat{A}|\varphi\rangle$ to $\widehat{\mathrm{BA}}|\varphi\rangle$, accordingly the co-spin quantum number $c$ is added, the derivation and description are as follows:

$$
\begin{gathered}
\hat{\mathrm{Q}} \widehat{\mathrm{B}}(\widehat{\mathrm{A}}|\varphi\rangle)-\widehat{\mathrm{B}} \widehat{\mathrm{Q}}(\widehat{\mathrm{A}}|\varphi\rangle)=c \widehat{\mathrm{B}}(\widehat{\mathrm{A}}|\varphi\rangle) \\
\widehat{\mathrm{Q}} \hat{\mathrm{B}} \widehat{\mathrm{A}}|\varphi\rangle=(c-q) \hat{\mathrm{B}} \widehat{\mathrm{A}}|\varphi\rangle
\end{gathered}
$$

Finally, the third state is transformed by $\widehat{\mathrm{C}}$ from $\widehat{\mathrm{B}} \widehat{A}|\varphi\rangle$ to $\widehat{\mathrm{C}} \widehat{\mathrm{BA}}|\varphi\rangle$, accordingly the rotation quantum number $r$ is added, the derivation and description are as follows:

$$
\begin{gathered}
\hat{\mathrm{Q}} \hat{\mathrm{C}}(\widehat{\mathrm{B}} \widehat{\mathrm{A}}|\varphi\rangle)-\hat{\mathrm{CQ}} \widehat{\mathrm{Q}}(\hat{\mathrm{B}} \widehat{\mathrm{A}}|\varphi\rangle)=r \widehat{\mathrm{C}}(\widehat{\mathrm{B}} \widehat{\mathrm{A}}|\varphi\rangle) \\
\hat{\mathrm{Q}} \hat{\mathrm{C}} \widehat{\mathrm{B}} \widehat{\mathrm{A}}|\varphi\rangle=(r+c-q) \hat{\mathrm{CB}} \widehat{\mathrm{A}}|\varphi\rangle
\end{gathered}
$$

Sequentially apply the inverse operators $\widehat{C}^{-1}, \widehat{B}^{-1}$, and $\widehat{A}^{-1}$ to the second term of Formula (5), which is reduced to the expression of transformed charge operator acting on the original eigenstate:

$$
\widehat{\mathrm{D}}|\varphi\rangle=(r+c-q)|\varphi\rangle, \widehat{\mathrm{D}}=\widehat{\mathrm{A}}^{-1} \widehat{\mathrm{B}}^{-1} \widehat{\mathrm{C}}^{-1} \widehat{\mathrm{Q}} \widehat{\mathrm{C}} \widehat{\mathrm{B}} \widehat{\mathrm{A}}
$$


What are the scopes of the eigenvalue of Formula (6)? These features depend on the operator properties, so the characteristics of operator $\widehat{D}$ should be analyzed. The square of operator $\hat{\mathrm{D}}$ is as follows:

$$
\widehat{D}^{2}=\widehat{A}^{-1} \widehat{B}^{-1} \widehat{C}^{-1} \widehat{Q} \widehat{C} \hat{B} \widehat{A} \widehat{A}^{-1} \widehat{B}^{-1} \widehat{C}^{-1} \widehat{Q} \hat{C} \widehat{B} \widehat{A}=\widehat{A}^{-1} \widehat{B}^{-1} \widehat{C}^{-1} \widehat{Q}^{2} \widehat{C} \widehat{B} \widehat{A}
$$

According to the expression of charge operator $\widehat{Q}$ in Formula (1): $\widehat{\mathrm{Q}}^{2}= \pm \widehat{\mathrm{Q}}$, so $\widehat{\mathrm{D}}^{2}$ can be simplified as:

$$
\widehat{D}^{2}= \pm \widehat{A}^{-1} \widehat{B}^{-1} \widehat{C}^{-1} \widehat{Q} \hat{\mathrm{C}} \widehat{B} \widehat{A}= \pm \widehat{D}
$$

The operator properties described in Formula (8) determine that the eigenvalue of Formula (6) is 0 or \pm 1 , which can be named as intrinsic sum of quantum numbers for physical particles, and its value determine the category of particles:

$$
r+c-q=0, \pm 1
$$

Clearly, $\hat{D}$ is the transformed result of $\widehat{Q}$ through interactions of physical operators, and Formula (6) describes the intrinsic properties of conservation, quantum and eigenstate of charges, so Formula (9) is the constrained equation of the quantum numbers $q, c$ and $r$ for physical particles. In Formula (9), adopting freedom mechanism of group theory can know that: when the intrinsic sum is 0 , the classification number of corresponding particles is $(2 \times 0+1)=1$, so hadrons justly satisfy this condition; when the intrinsic sum is \pm 1 , the absolute value of the intrinsic sum is 1 , so the classification number of corresponding particles is $(2 \times 1+1)=3$, leptons justly satisfy this condition.

\subsection{Equation Expression by Classical Quantum Numbers}

Using Formula (9) to describe the quantum number constrained equation for physical particles, quantum numbers $q, c$ and $r$ are closely related to classical quantum numbers. Respectively, $q$ is the classical charge quantum number; Co-spin quantum number $c$ describes the comprehensive effect of particle classification and spin state, so which is defined as the product of spin quantum number and sign of positive or antiparticle particles, and the relevant classical value is half of the baryon number $b$ and the lepton number $l$ :

$$
c=b / 2 \text { (for hadrons), } c=l / 2 \text { (for leptons) }
$$

Rotation transformation is similar to isospin transformation, can be used to describe the exchange effect between singularity and isospin, so rotation quantum number $r$ is defined as:

$$
r=s / 2+i_{3}
$$

Where $s$ is singular number, $i_{3}$ is third isospin component.

As the result, using $\left(s, i_{3}, \mathrm{c}, q\right)$ as the conventional quantum numbers of physical particles, the expression of Formula (9) is:

$$
s / 2+i_{3}+c-q=0, \pm 1
$$

\subsection{Quantum Number Constrained Equation for Hadrons or Leptons}

When the intrinsic sum is 0 , Formula (12) is the quantum number constrained equation for hadrons, namely:

$$
s / 2+i_{3}+b / 2-q=0
$$

Obviously, this expression is completely consistent with Gell-Mann-Nishijima formula. Use the existing data to verify, indicating that Formula (13) is right for hadrons, for examples: baryons (p, n), mesons $\left(\pi^{+}, \pi^{0}, \pi^{-}, \mathrm{k}^{+}, \mathrm{k}^{0}, \mathrm{k}^{-}\right)$and hyperons $\left(\Lambda, \Sigma^{+}, \Sigma^{0}, \Sigma^{-}, \Xi^{0}, \Xi^{-}\right)$, etc. In addition, the classification of hadrons can also be explained: in Formula (13), the intrinsic sum is 0 , and adopt freedom mechanism of group theory, can know that the number of hadron classification is $(2 \times 0+1)=1$, which indicating: all hadrons belong to the same class, so the conservation of baryon number is not classified by category.

When the intrinsic sum is 1 or -1 , Formula (12) is the quantum number constrained equation for leptons, namely:

$$
s / 2+i_{3}+l / 2-q= \pm 1
$$

Where, lepton singular number $s$ is introduced in order to generally descript the expression and predict the production of new leptons. From Formula (14), three rules of leptons can be revealed: (1) Distinguishing particle and antiparticle by intrinsic sum: when intrinsic sum is 1 , the leptons are positive particles; when the intrinsic sum is -1 , the leptons are antiparticles. For a pair of particle and antiparticles, of each other the quantum numbers and intrinsic sum are opposite in sign and equal in value. (2) The lepton meson with spin 0 does not exist, for that when $c=0$ the quantum numbers in Formula (14) have no reasonable solutions. (3) Lepton classification: the absolute value of the intrinsic sum is 1 , so the number of lepton classification is $(2 \times 1+1)=3$, indicating that: there are 3 classes of leptons, so when the leptons belong to different classes, the nonzero Co-spin numbers cannot be summed, and the conservation of lepton numbers must be classified by category. At present, there are 3 kinds of leptons, the properties and quantum numbers are completely consistent with this theory (Refer to Table 1).

Table 1. Lepton quantum numbers.

\begin{tabular}{lllll}
\hline Quantum number & $\mathbf{e}^{-}, \boldsymbol{\mu}^{-}, \tau^{-}$ & $\boldsymbol{v}_{\mathbf{e}}, \boldsymbol{v}_{\boldsymbol{\mu}}, \boldsymbol{v}_{\boldsymbol{\tau}}$ & $\mathbf{e}^{+}, \boldsymbol{\mu}^{+}, \tau^{+}$ & $\overline{\boldsymbol{v}}_{\mathbf{e}}, \overline{\boldsymbol{v}}_{\boldsymbol{\mu}}, \overline{\boldsymbol{v}}_{\boldsymbol{\tau}}$ \\
\hline$q$ & -1 & 0 & +1 & 0 \\
$c$ & $1 / 2$ & $1 / 2$ & $-1 / 2$ & $-1 / 2$ \\
$i_{3}$ & $-1 / 2$ & $1 / 2$ & 0 & $-1 / 2$ \\
$s$ & 0 & 0 & -1 & 0 \\
Intrinsic sum & +1 & +1 & -1 & \\
\hline
\end{tabular}




\section{Singular Number Judgment of Singular Particles}

\subsection{Virtual Structure Hypothesis}

Singular particles must be composite particles, therefore we can study the properties of singular particles from the composition and structure of composite particles. A particle can decay into more than one particle, the particle is called composite particle. Some of the particles produced by decay are also composite particles, so they also decay to produce other particles. In this way, through gradual decay, the final products will be some relatively stable basic particles, such as electrons, neutrons, protons, neutrinos, etc. All the basic particles produced by the gradual decay of composite particles are called the component particles of the first composite particle. A composite particle is constructed by its component particles by forming corresponding structure, this hypothesis structure can be called virtual structure of composite particles, which has a great influence on the properties of composite particle.

C-N pair assumption: as the components of a composite particle, charged elementary particle $x$ and its neutral antiparticle $\bar{v}_{x}$ may form pair $\left[x, \bar{v}_{x}\right]$ when they meet and be reconciled by other component particles in parent particle, so the quantum number of the pair is $(0,-1,0,-1)$ or $(0,1,0,1)$. The pair mainly change the charge value and part mass of composite particle. Here use $\mathrm{C}-\mathrm{N}$ pair as the abbreviation.

P-A pair assumption: elementary particle $x$ and its antiparticle $\bar{x}$, will annihilate when they meet alone; however, if as the components of a composite particle, may form pair $\{x, \bar{x}\}$ when they meet and be reconciled by other component particles in parent particle, so the quantum number of the pair is $(0,0,0,0)$. Here use P-A pair as the abbreviation. Physically, P-A pairs are formed due to the interaction force between particles and antiparticles, so the mass of the composite particle will be very large when there are P-A pairs formed in its component particle swarm.

Locator assumption: in the component particles of a composite particle, there must be an elementary particle which belongs to the same class with composite particle, thus has the same Co-spin quantum number $c$ with composite particle and determines the categorical properties of composite particle. Therefore, this component particle is called locator in virtual structure of composite particle. Specifically, consider $\mathrm{e}^{-}, v_{\mathrm{e}}$, $v_{\mu}$ and $v_{\tau}$ as lepton locators, consider $\mathrm{p}$ and $\mathrm{n}$ as baryon locators, and suppose quark pair $\mathrm{o}=(\mathrm{u} \overline{\mathrm{u}})$ as meson locator with quantum number $(0,0,0,0)$.

Virtual structure assumption: A composite particle is composed of several component particles, being similar to that a family is made up by several peoples. In the component particles, some form C-N pairs, some form P-A pairs, and one acts as the locator. In this way, a composite particle has a virtual structure being consist of C-N pairs, P-A pairs and a locator, so its properties are closely related to the number of $\mathrm{C}-\mathrm{N}$ pairs and P-A pairs, and the nature of the locator.
Drawing analogy with human family structure, C-N pair is analogue to brother-sister pair, P-A pair is analogue to husband-wife pair, and locator is analogue to patriarch.

\subsection{Singular Number Calculation}

Conserved quantum numbers between composite and component particles: In the intrinsic space, inversion transformation, co-spin transformation and rotation transformation are symmetric transformations, so these three symmetry transformations do not change the conservation of quantum numbers $q, c$ and $r$. The conservation rule of quantum numbers is mainly reflected in the process of decay and construction of composite particles. Suppose that the quantum numbers of a composite particle are $q, c$ and $r$, and the sum of term by term quantum numbers of the component particles are $q^{\prime}, c^{\prime}$ and $r^{\prime}$. According to the law of conservation, the quantum number of a composite particle is equal to the sum of the quantum numbers of the component constituent particles, namely:

$$
q^{\prime}=q, c^{\prime}=c, r^{\prime}=r
$$

The generating mechanism and calculation method of singular number: According to the previous assumptions, P-A pairs may produce a lot of mass, and thus make the composite particles have singularity and large mass. Therefore, a theoretical explanation is proposed, namely: singularity is produced by P-A pairs, so the singular number is the number of P-A pairs. The conservation and variation of quantum numbers between component particles and composite particle are expressed by Formula (15), so which can be used to calculate the quantum numbers of composite particle. In the component particles, there is no singularity, so the rotation quantum number $r^{\prime}$ of component particles is only the third component of isospin $i_{3}{ }^{\prime}$, namely $r^{\prime}=i_{3}{ }^{\prime}$. For composite particle, if there are P-A pairs in the virtual structure, singular number $s$ is generated and contribute to the rotation quantum number $r$, namely $r=s / 2+i_{3}$. According to the Formula (15), the quantum number change results related to the singular number can be obtained:

$$
s / 2+i_{3}=i_{3}^{\prime},|s|=n_{\mathrm{p}}
$$

Where $n_{\mathrm{p}}$ is the total number of $\mathrm{P}$-A pairs in virtual structure. Based on Formula (16), the singular number can be calculated, and the non-conservation phenomenon of singular numbers can be reasonably explained. For instance, the disappearance of the P-A pairs after the decay of the singular particle can result in the disappearance of the singular number, indicating the reason of the non-conservation of singular numbers.

\subsection{Example Verification}

(1) Lepton $\tau^{-}$may has singularity

For lepton $\tau^{-}: \tau^{-} \rightarrow \mu^{-}+\bar{v}_{\mu}+v_{\tau}, \mu^{-} \rightarrow \mathrm{e}^{-}+\bar{v}_{\mathrm{e}}+v_{\mu}$, so 
the component particles are $\left(\mathrm{e}^{-}, \bar{v}_{\mathrm{e}}, v_{\mu}, \bar{v}_{\mu}, v_{\tau}\right)$, and the sum of quantum numbers of component particles is $(0,-1 / 2,1 / 2$, -1). There are two possible virtual structures: the first one may be $\left[\mu^{-}, \bar{v}_{\mu}\right]+v_{\tau} \rightarrow \tau^{-}$, of which there is no P-A pair, so the corresponding quantum number is $(0,-1 / 2,1 / 2,-1)$; the second one may be $\left[\mathrm{e}^{-}, \bar{v}_{\mathrm{e}}\right]+\left\{v_{\mu}, \bar{v}_{\mu}\right\}+v_{\tau} \rightarrow \tau^{-}$, of which there is a P-A pair, so take $n_{\mathrm{p}}=1$ and $i_{3}^{\prime}=-1 / 2$ into Formula (16), and refer to the quantum number conservation mechanism descripted by Formula (15), the quantum number of the composite particles with this second structure is $(-1,0,1 / 2,-1)$. Which structure belongs to the current lepton $\tau^{-}$? The mass of $\tau^{-}$is very large: $1776.82 \mathrm{MeV}$. The existence of P-A pair can produce large mass, so the second virtual structure may belong to $\tau^{-}$, providing singular number -1 . To prove this assumption, further experiments needed. If a new lepton of the same family as $\tau^{-}$is found through experiments, the structure of $\tau^{-}$is the first one when the mass of the new lepton is greater than $1776.82 \mathrm{MeV}$, or the structure of $\tau^{-}$is the second one when the mass of the new lepton is less than $1776.82 \mathrm{MeV}$.

(2) Mesons $\pi^{-}$and $\mathrm{k}^{-}$have the same components, but the mass are very different, why?

For mesons $\pi^{-}$and $\mathrm{k}^{-}: \pi^{-} \rightarrow \mu^{-}+\bar{v}_{\mu}, \mathrm{k}^{-} \rightarrow \mu^{-}+\bar{v}_{\mu}$, showing that the component particles of $\pi^{-}$and $\mathrm{k}^{-}$are the same $\left(\mathrm{e}^{-}, \bar{v}_{\mathrm{e}}, v_{\mu}, \bar{v}_{\mu}, \mathrm{o}\right)$, and the sum of quantum numbers of component particles is $(0,-1,0,-1)$. There are two possible virtual structures: the first one generate $\pi^{-}$, namely $\left[\mu^{-}, \bar{v}_{\mu}\right]+o \rightarrow \pi^{-}$, of which there is no P-A pair, so the corresponding quantum number is $(0,-1,0,-1)$, which is just the quantum number of $\pi^{-}$. The second one generate $\mathrm{k}^{-}$, namely $\left[\mathrm{e}^{-}, \bar{v}_{\mathrm{e}}\right]+\left\{v_{\mu}, \bar{v}_{\mu}\right\}+\mathrm{o} \rightarrow \mathrm{k}^{-}$, of which there is $1 \mathrm{P}-\mathrm{A}$ pair, so take $n_{\mathrm{p}}=1$ and $i_{3}^{\prime}=-1$ into Formula (16) and (15), the quantum number of the composite particle with the second structure is $(-1,-1 / 2,0,-1)$. The quantum number is just equal to that of $\mathrm{k}^{-}$, proving that this structure is the composition mode of $\mathrm{k}^{-}$. The masses of $\pi^{-}$and $\mathrm{k}^{-}$are respectively $139.57 \mathrm{MeV}$ and $493.677 \mathrm{MeV}$, and the latter is greater than the former, implying that there is a $\mathrm{P}$-A pair in $\mathrm{k}^{-}$, no $\mathrm{P}$-A pair in $\pi^{-}$. Under the condition of the same component particles, the difference of mass and third isospin component between $\mathrm{k}^{-}$ and $\pi^{-}$, prove that it is reasonable to explain the singular number by the concept of P-A pairs.

(3) Hyperons $\Lambda, \Xi^{-}$and $\Omega^{-}$have different singular numbers

For hyperon $\Lambda: \Lambda \rightarrow p+\pi^{-}$, so the component particles are $\left(\mathrm{p}, \mathrm{e}^{-}, \bar{v}_{\mathrm{e}}, v_{\mu}, \bar{v}_{\mu}\right)$, and the sum of quantum numbers of component particles is $(0,-1 / 2,1 / 2,0)$. The virtual structure is $\mathrm{p}+\left[\mathrm{e}^{-}, \bar{v}_{\mathrm{e}}\right]+\left\{v_{\mu}, \bar{v}_{\mu}\right\} \rightarrow \Lambda$, of which there is a $\mathrm{P}-\mathrm{A}$ pair, so take $n_{\mathrm{p}}=1$ and $i_{3}^{\prime}=-1 / 2$ into Formula (16) and (15), the quantum number of this structure is $(-1,0,1 / 2,0)$, which is just equal to that of $\Lambda$, proving that this structure is the composition mode of $\Lambda$, singular number is -1 .

For hyperon $\Xi^{-}: \Xi^{-} \rightarrow \Lambda+\pi^{-} \rightarrow \mathrm{p}+\pi^{-}+\pi^{-}$, so the component particles are $\left(\mathrm{p}, 2 \mathrm{e}^{-}, 2 \bar{v}_{\mathrm{e}}, 2 v_{\mu}, 2 \bar{v}_{\mu}\right)$, and the sum of quantum numbers of component particles is $(0,-3 / 2$, $1 / 2, \quad-1)$. The virtual structure is $\mathrm{p}+2\left[\mathrm{e}^{-}, \bar{v}_{\mathrm{e}}\right]+2\left\{v_{\mu}, \bar{v}_{\mu}\right\} \rightarrow \Xi^{-}$, of which there are $2 \mathrm{P}-\mathrm{A}$ pairs, so take $n_{\mathrm{p}}=2$ and $i_{3}^{\prime}=-3 / 2$ into Formula (16) and (15), the quantum number of this structure is $(-2,-1 / 2,1 / 2,-1)$, which is just equal to that of $\Xi^{-}$, proving that this structure is the composition mode of $\Xi^{-}$, singular number is -2 .

For hyperon $\Omega^{-}: \Omega^{-} \rightarrow \Lambda+\mathrm{k}^{-} \rightarrow \mathrm{p}+\pi^{-}+\mathrm{k}^{-}$, $\mathrm{p} \rightarrow \mathrm{n}+\mathrm{e}^{+}+v_{\mathrm{e}}$ and $\pi^{-} \rightarrow \mathrm{e}^{-}+\bar{v}_{\mathrm{e}}$, so the component particles are (n, $\left.2 \mathrm{e}^{-}, 2 \bar{v}_{\mathrm{e}}, \mathrm{e}^{+}, v_{\mathrm{e}}, v_{\mu}, \bar{v}_{\mu}\right)$, and the sum of quantum numbers of component particles is $(0,-3 / 2,1 / 2,-1)$. The virtual structure is $\mathrm{n}+\left[\mathrm{e}^{-}, \bar{v}_{\mathrm{e}}\right]+\left\{\mathrm{e}^{-}, \mathrm{e}^{+}\right\}+\left\{v_{\mathrm{e}}, \bar{v}_{\mathrm{e}}\right\}+\left\{v_{\mu}, \bar{v}_{\mu}\right\} \rightarrow \Omega^{-}$, of which there are $3 \mathrm{P}$-A pairs, so take $n_{\mathrm{p}}=3$ and $i_{3}=-3 / 2$ into Formula (16) and (15), the quantum number of this structure is $(-3,0,1 / 2,-1)$, which is just equal to that of $\Omega^{-}$, proving that this structure is the composition mode of $\Omega^{-}$, singular number is -3 .

(4) The selection rule of singular number change

About hyperon $\Xi^{0}$, why is $\Xi^{0} \rightarrow \Lambda+\pi^{0}$ always the observed decay, but not $\Xi^{0} \rightarrow \mathrm{n}+\pi^{0}$ ? This question can be answered by analyzing the virtual structure synthesized by component particles. If $\Xi^{0} \rightarrow \Lambda+\pi^{0}$, the component particles are $\left(\mathrm{p}, 2 \mathrm{e}^{-}, \mathrm{e}^{+}, \bar{v}_{\mathrm{e}}, v_{\mu}, \bar{v}_{\mu}\right)$, so virtual structure is $\mathrm{p}+\left[\mathrm{e}^{-}, \bar{v}_{\mathrm{e}}\right]+\left\{\mathrm{e}^{-}, \mathrm{e}^{+}\right\}+\left\{v_{\mu}, \bar{v}_{\mu}\right\} \rightarrow \Xi^{0}$, of which there are 2 P-A pairs, thus the singular number is -2 . If $\Xi^{0} \rightarrow \mathrm{n}+\pi^{0}$, the component particles are ( $\left.\mathrm{p}, 2 \mathrm{e}^{-}, \mathrm{e}^{+}, \bar{v}_{\mathrm{e}}\right)$, so virtual structure is $\mathrm{p}+\left[\mathrm{e}^{-}, \bar{v}_{\mathrm{e}}\right]+\left\{\mathrm{e}^{-}, \mathrm{e}^{+}\right\} \rightarrow \Sigma^{0}$, of which there are $1 \mathrm{P}-\mathrm{A}$ pair, thus the singular number is -1 , and the quantum number is $(-1$, $0,1 / 2,0)$, which is not equal to that of $\Xi^{0}$, indicating that decay $\Xi^{0} \rightarrow \mathrm{n}+\pi^{0}$ can't occur from $\Xi^{0}$.

About hyperon $\Xi^{-}$, why is $\Xi^{-} \rightarrow \Lambda+\pi^{-}$always the observed decay, but not $\Xi^{-} \rightarrow \mathrm{n}+\pi^{-}$? This question can be answered by analyzing the virtual structure synthesized by component particles. If $\Xi^{-} \rightarrow \Lambda+\pi^{-}$, the component particles are ( $\left.\mathrm{p}, 2 \mathrm{e}^{-}, 2 \bar{v}_{\mathrm{e}}, 2 v_{\mu}, 2 \bar{v}_{\mu}\right)$, so virtual structure is 
$\mathrm{p}+2\left[\mathrm{e}^{-}, \bar{v}_{\mathrm{e}}\right]+2\left\{v_{\mu}, \bar{v}_{\mu}\right\} \rightarrow \Xi^{-}$, of which there are $2 \mathrm{P}-\mathrm{A}$ pairs, thus the singular number is -2 . If $\Xi^{-} \rightarrow n+\pi^{-}$, the component particles are $\left(\mathrm{p}, 2 \mathrm{e}^{-}, 2 \bar{v}_{\mathrm{e}}, v_{\mu}, \bar{v}_{\mu}\right)$, so virtual structure is $\mathrm{p}+2\left[\mathrm{e}^{-}, \bar{v}_{\mathrm{e}}\right]+\left\{v_{\mu}, \bar{v}_{\mu}\right\} \rightarrow \Sigma^{-}$, of which there is 1 $\mathrm{P}-\mathrm{A}$ pair, thus the singular number is -1 , and the quantum number is $(-1,-1,1 / 2,-1)$, indicating that decay $\Xi^{-} \rightarrow \mathrm{n}+\pi^{-}$ can't occur from $\Xi^{-}$.

\subsection{New Particle Prediction}

(1) A new hyperon $X$ with $(-1,-1,1 / 2,-1)$ and $X \rightarrow \Lambda+\pi^{-}$ may exist

Suppose a particle $\mathrm{X}$ is found in experiments, and its decay is: $\mathrm{X} \rightarrow \Lambda+\pi^{-}$, how many possible kinds are there for this particle? For that $\Lambda \rightarrow \mathrm{p}+\pi^{-}, \pi^{-} \rightarrow \mu^{-}+\bar{v}_{\mu} \quad$ or $\pi^{-} \rightarrow \mathrm{e}^{-}+\bar{v}_{\mathrm{e}}$, so the virtual structure of the particle $\mathrm{X}$ will be different depending on the structure of $\pi^{-}$. The first possibility: accept $\pi^{-} \rightarrow \mu^{-}+\bar{v}_{\mu}$ for all, the virtual structure may be $\mathrm{p}+2\left[\mathrm{e}^{-}, \bar{v}_{\mathrm{e}}\right]+2\left\{v_{\mu}, \bar{v}_{\mu}\right\} \rightarrow \mathrm{X}$, and the quantum number is $(-2,-1 / 2,1 / 2,-1)$, so under this condition particle $\mathrm{X}$ just is hyperon $\Xi^{-}$. The second possibility: accept $\pi^{-} \rightarrow \mu^{-}+\bar{v}_{\mu}$ in $\Lambda \rightarrow \mathrm{p}+\pi^{-}$, and accept $\pi^{-} \rightarrow \mathrm{e}^{-}+\bar{v}_{\mathrm{e}}$ in $\mathrm{X} \rightarrow \Lambda+\pi^{-}$, so the component particles are (p, $2 \mathrm{e}^{-}, 2 \bar{v}_{\mathrm{e}}$, $\left.v_{\mu}, \bar{v}_{\mu}\right)$, and the virtual structure may be $\mathrm{p}+2\left[\mathrm{e}^{-}, \bar{v}_{\mathrm{e}}\right]+\left\{v_{\mu}, \bar{v}_{\mu}\right\} \rightarrow \mathrm{X}$. For this structure, the sum of quantum numbers of component particles is $(0,-3 / 2,1 / 2,-1)$, and there is $1 \mathrm{P}-\mathrm{A}$ pair, so take $n_{\mathrm{p}}=1$ and $i_{3}^{\prime}=-3 / 2$ into Formula (16) and (15), the quantum number of this structure is $(-1,-1,1 / 2,-1)$. The quantum numbers is equal to that of $\Sigma^{-}$, however the decay of $\Sigma^{-}$is $\Sigma^{-} \rightarrow \mathrm{n}+\pi^{-}$, which is not as the decay of $X$. It shows that there exists a new kind of particle $\mathrm{X}$, its quantum number is $(-1,-1,1 / 2,-1)$, and its decay is $\mathrm{X} \rightarrow \Lambda+\pi^{-}$, expecting experiments to verify the prediction of this new particle. Note: Compared with $\Sigma^{-}$, the mass of $\mathrm{X}$ is less than that of $\Sigma^{-}$.

(2) A new hyperon $\mathrm{Y}$ with $(-4,1 / 2,1 / 2,-1)$ and $\mathrm{Y} \rightarrow \Lambda+\mathrm{k}^{-}$may exist

Suppose new particle $\mathrm{Y}$ is found in experiments, and its decay is: $\mathrm{Y} \rightarrow \Lambda+\mathrm{k}^{-}$, how many possible kinds are there for this particle? For that $\Lambda \rightarrow \mathrm{p}+\pi^{-}, \pi^{-} \rightarrow \mu^{-}+\bar{v}_{\mu}$ or $\pi^{-} \rightarrow \mathrm{e}^{-}+\bar{v}_{\mathrm{e}}$, so the virtual structure of particle $\mathrm{Y}$ may be different depending on the virtual structure of $\pi^{-}$. The first possibility: accept $\pi^{-} \rightarrow \mathrm{e}^{-}+\bar{v}_{\mathrm{e}}$, it has been proved that the particle $\mathrm{Y}$ is hyperon $\Omega^{-}$. The second possibility: accept $\pi^{-} \rightarrow \mu^{-}+\bar{v}_{\mu} \quad, \quad$ the virtual structure is $\mathrm{n}+\left[\mathrm{e}^{-}, \bar{v}_{\mathrm{e}}\right]+\left\{\mathrm{e}^{-}, \mathrm{e}^{+}\right\}+\left\{v_{\mathrm{e}}, \bar{v}_{\mathrm{e}}\right\}+2\left\{v_{\mu}, \bar{v}_{\mu}\right\} \rightarrow \mathrm{Y}$. For this structure, the sum of quantum numbers of component particles is $(0,-3 / 2,1 / 2,-1)$, and there are 4 P-A pairs, so take $n_{\mathrm{p}}=4$ and $i_{3}^{\prime}=-3 / 2$ into Formula (16) and (15), the quantum number of this structure is $(-4,1 / 2,1 / 2,-1)$. In this quantum number, the signs of the third component of isospin and the charge are not same, which is unreasonable in nonsingular particles; however, if the singular number is equivalent to the third component of isospin, this quantum number is reasonable. At present, the quantum number is $(-4,1 / 2,1 / 2$, -1 ), and the decay is $\mathrm{Y} \rightarrow \Lambda+\pi^{-}$, particle $\mathrm{Y}$ consistent with this data has not been found. It is hoped that someone can verify this new particle prediction through experiments. Note: Compared with $\Omega^{-}$, the mass of Y may be greater than that of $\Omega^{-}$.

\section{Summary}

The quantum number constrained equation of physical particles is established theoretically, and an effective explanation is given for the generation of singularities and the calculation of singular numbers. The theoretical results in this paper are applicable to physical particles, and the predictions of lepton singularity and new singular hadrons are also valuable. In order to further verify this theory and promote particle physics, I found that the following questions need to be considered deeply: The quantum number constrained equation can be used to describe hadrons and leptons, is there a limit to the scope of application? As a hypothesis, the particle-antiparticle pair can introduce singularity, how to explain it from the physical mechanism? Vector field particles, such as photons, gluons, and weakly interacting bosons, how to express the quantum number constrained equations? It is hoped that physics peers positively think about the above problems in theory, and verify the ideas proposed in this paper from experiments, so as to promote the perfect development of particle physics.

\section{Acknowledgements}

The author is grateful to the support from the National Natural Science Foundation of China (Grant No. 61671467), and the Innovation Team of Shaanxi province (Grant No. 2014KCT-05).

\section{References}

[1] D. Morgan and M. R. Pennington, Pole Structures in Nuclear and Particle Physics, Phys. Rev. Lett. Vol. 59: 2818 (1987).

[2] Leon M. Lederman, Observations in Particle Physics from Two Neutrinos to the Standard Model, Science Vol. 244: 4905 (1989).

[3] Hans Dehmelt, Experiments on the Structure of an Individual Elementary Particle, Science Vol. 247: 4942 (1990). 
[4] David D. Mille, John M. Doyle, Alexander O. Sushkov, Probing the frontiers of particle physics with tabletop-scale experiments, Science Vol. 357: 6355 (2017).

[5] Andrea Vanossi, Clemens Bechinger, Michael Urbakh, Structural lubricity in soft and hard matter systems, Nature Communications Vol. 11: 4657 (2020).

[6] Pushpalatha C. Bhat, Geoffrey N. Taylor, Particle physics at accelerators in the United States and Asia, Nature Physics Vol. 16: 380-385 (2020).

[7] Ramón Ramos, David Spierings, Isabelle Racicot \& Aephraim M. Steinberg, Measurement of the time spent by a tunnelling atom within the barrier region, Nature Vol. 583: 529-532 (2020).

[8] T. Terasawa, M. Scholer, The Heliosphere as an Astrophysical Laboratory for Particle Acceleration, Science Vol. 244: 4908 (1989).

[9] E. L. Chupp, Transient Particle Acceleration Associated with Solar Flares, Science Vol. 250: 4978 (1990).

[10] John Ellis \& Ian Wilson, New physics with the Compact Linear Collider, Nature Vol. 409: 431-435 (2001).

[11] James J. De Yoreo, Pupa U. P. A. Gilbert, Nico A. J. M. Sommerdijk, R. Lee Penn, et. al. Crystallization by particle attachment in synthetic, biogenic, and geologic environments, Science Vol. 349: 6247 (2015).

[12] J. Wang, S. Bose, C. S. Frenk, L. Gao, A. Jenkins, V. Springel, S. D. M. White, Universal structure of dark matter haloes over a mass range of 20 orders of magnitude, Nature Vol. 585: 39$42(2020)$.
[13] Mingyi Wang, Weimeng Kong, Neil M. Donahue, Rapid growth of new atmospheric particles by nitric acid and ammonia condensation, Nature Vol. 581: 184-189 (2020).

[14] Chris Q, Roy F, Elementary Particle Physics and the Superconducting Super Collider, Science Vol. 231: 4745 (1986).

[15] A. Pais, Theoretical particle physics, Rev. Mod. Phys. Vol. 71: 16 (1999).

[16] Mary K. Gaillard, Paul D. Grannis, and Frank J. Sciulli, The standard model of particle physics, Rev. Mod. Phys. Vol. 71: 96 (1999).

[17] F. Archilli, M.-O. Bettler, P. Owen \& K. A. Petridis, Flavour-changing neutral currents making and breaking the standard model, Nature Vol. 546; 221-226 (2017).

[18] Joerg Jaeckel, Mike Lamont, Claude Vallée, The quest for new physics with the Physics Beyond Colliders program, Nature Physics Vol. 16: 393-401 (2020).

[19] Lee T. D, Particle Physics and Introduction to Field Theory (Harwood-Academic Publishers, ISBN: 978-3-71-860033-5, 1981).

[20] Zhang Naisen, Particle Physics (Science Press, ISBN: 978-7-03-004205-7, 1984).

[21] Lu Tan, Luo Liaofu, Exploration of matter: from Electrons to Quarks (Science Press, ISBN: 978-7-03-047726-2, 2016).

[22] Zhu Hongyuan, Group theory and Symmetry in Quantum mechanics (Peking University Press, ISBN: 978-7-301-14547-0, 2009). 